\title{
The Lorentz angle in silicon detectors
}

\author{
W. de Boer*, V. Bartsch, J. Bol, A. Dierlamm, E.Grigoriev, F. Hauler, L. Jungermann \\ IEKP, Univ. of Karlsruhe, Postfach 6980, 76128 Karlsruhe, Germany \\ E-mail: 'wim.de.boer@cern.ch'
}

ABSTRACT: Future detectors will use silicon sensors in the harsh radiation environment of the LHC (Large Hadron Collider) and high magnetic fields. The drift direction of the charge carriers is affected by the Lorentz force due to the high magnetic field. Also the resulting radiation damage changes the properties of the drift. This note describes the measurements of the Lorentz angle and a simple algorithm to calculate it as function of bias voltage, magnetic field and temperature.

\section{Introduction}

The Lorentz angle $\Theta_{\mathrm{L}}$, by which charge carriers are deflected in a magnetic field perpendicular to the electric field, is defined by:

$$
\tan \left(\Theta_{\mathrm{L}}\right)=\frac{\Delta x}{d}=\mu_{\mathrm{H}} B=r_{\mathrm{H}} \mu B
$$

where $d$ corresponds to the drift distance along the electric field and $\Delta x$ to the shift of the signal position. If the ionization is produced at the surface the drift distance equals the detector thickness. If the ionization is produced homogeneously by a traversing particle, the averaged drift distance is only half the detector thickness. The Hall mobility is denoted by $\mu_{\mathrm{H}}$, the conduction mobility by $\mu$. The Hall mobility differs from the conduction mobility by the Hall scattering factor $r_{\mathrm{H}}$. This factor describes the influence of the magnetic field on the mean free path of carriers of different energy [i]. The Hall scattering factor has a value

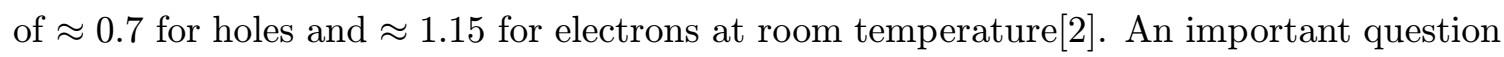
is the dependence of the Lorentz shift on the irradiation dose. The radiation changes the effective doping concentration and correspondingly the bias voltage for full depletion, as shown in Fig. iㅣ. This in turn changes the electric field in the detector and thus the Lorentz angle.

\footnotetext{
${ }^{*}$ Speaker.
} 


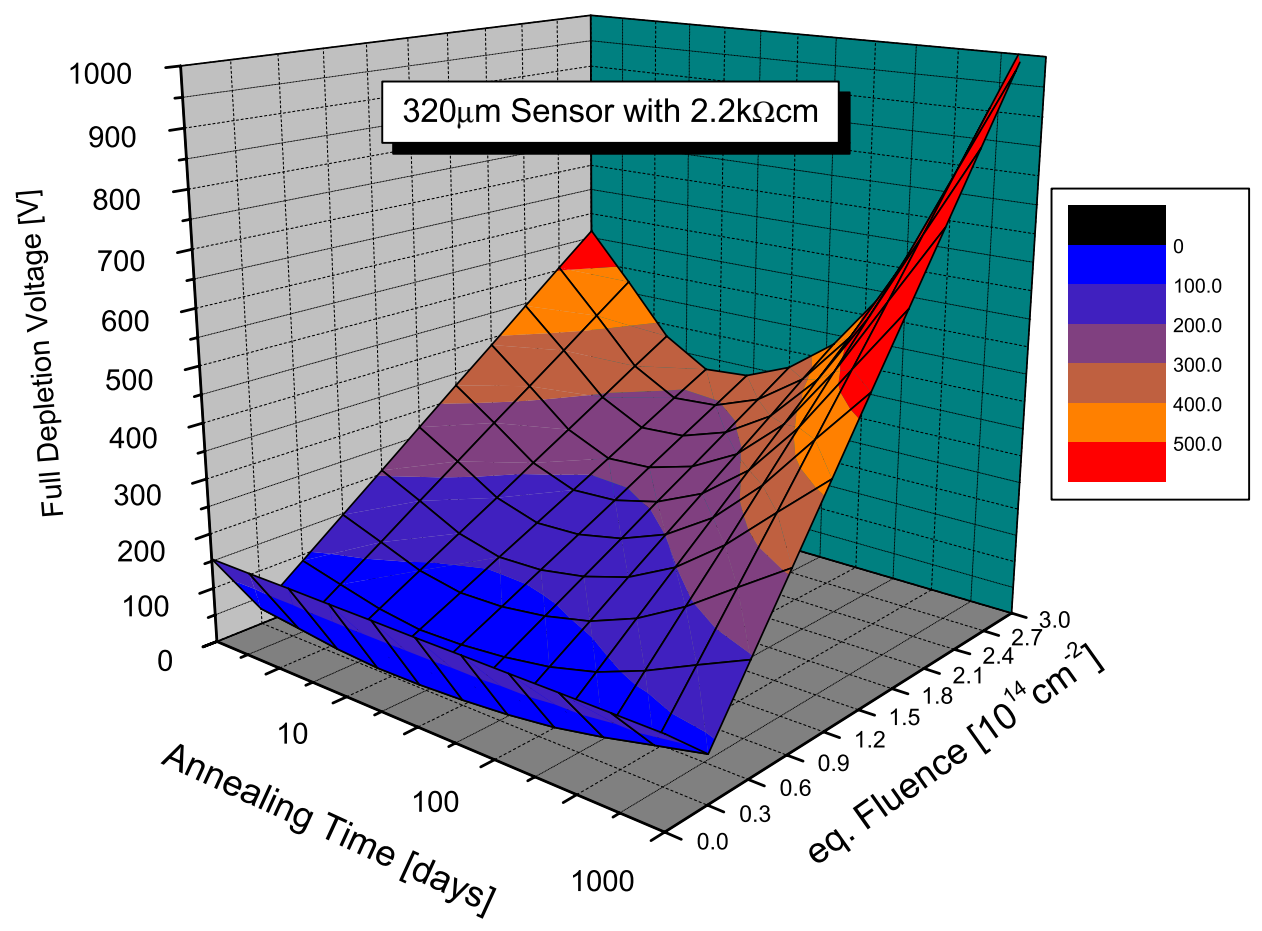

Figure 1: Bias voltage for full depletion as function of $1 \mathrm{MeV}$-equivalent neutron fluences and annealing time for inner and outer barrel sensors. The lines on the curved surface for a constant fluence show first the decrease in bias voltage due to the beneficial annealing followed by the reverse annealing. The different shades indicate the regions for a given bias voltage. The initial decrease in bias voltage for increasing fluences is due to the reduction of the effective doping concentration during the transition from $\mathrm{n}$ - to $\mathrm{p}$-doped material. The calculations were performed with the 'Hamburg model' [i़in.

\section{Experimental results}

A comprehensive study of Lorentz shifts of non-irradiated and irradiated sensors has been performed at Karlsruhe $\left[\begin{array}{l}{[4} \\ 1\end{array}\right]$. The Lorentz angle is measured by injecting red laser light at the surface on one side and measuring the position of the generated charge on the opposite side. The penetration of the red laser light with a wavelength of $660 \mathrm{~nm}$ and a maximum power of about $1 \mathrm{~mW}$ is a few $\mu \mathrm{m}$ at room temperature.

For the measurements the JUMBO magnet from the Forschungszentrum Karlsruhe [5] was used in a $B \leq 10 \mathrm{~T}$ configuration with a warm bore of $72 \mathrm{~mm}$. The sensors are double sided "baby" detectors of approximately $2 \mathrm{x} 1 \mathrm{~cm}$ from the HERA-B production by Sintef. They have a strip pitch of $50 \mu \mathrm{m}$ on the $\mathrm{p}$-side and $80 \mu \mathrm{m}$ on the $\mathrm{n}$-side; the strips 

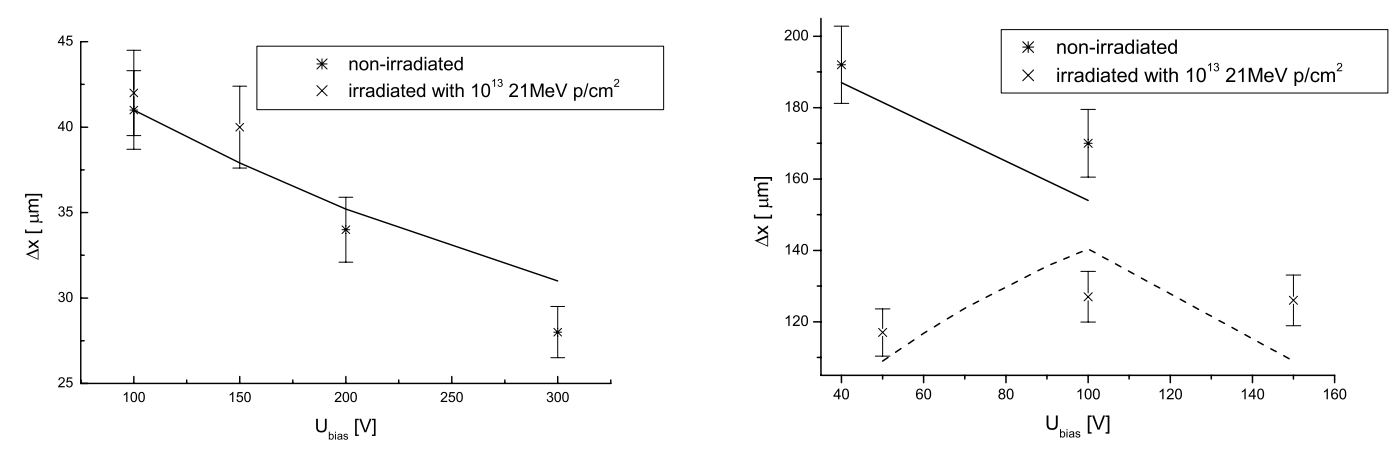

Figure 2: The Lorentz shift $\Delta x$ for holes (left) and electrons (right) as function of the bias voltage. The shift corresponds to a $4 \mathrm{~T}$ magnetic field and a drift distance of the laser-induced surface charge in the detector of $300 \mu m$. The curves are the result of the simulation. The reduced Lorentz shift for the irradiated sample below $100 \mathrm{~V}$ is due to the fact that the detector is not fully depleted, since the full depletion voltage changed from $40 \mathrm{~V}$ before to $100 \mathrm{~V}$ after irradiation.

on opposite sides are oriented at an angle of $90^{\circ}$ with respect to each other. The signal position is computed using either a fit with the sum of two Gaussians or the centre of gravity.

Before irradiation the detector depletes fully at a bias voltage of $40 \mathrm{~V}$, while after irradiation with $1.0 \cdot 10^{13} 21 \mathrm{MeV}$ protons / $\mathrm{cm}^{2}$ the depletion voltage has increased to $100 \mathrm{~V}$. This implies that the bulk is inverted from n-type to p-type material, as expected [市. The bulk damage of $21 \mathrm{MeV}$ protons is about 2.1 times the damage from $1 \mathrm{MeV}$ neutrons [i]. Numerical results on the Lorentz angles and shifts have been summarized in Fig. $\overline{2}$ ind Tab. ${ }_{-1}^{1}$ for irradiated and non-irradiated sensors. For holes the Lorentz angle is not strongly dependent on irradiation, while for electrons there is a clear dependence. The simulated Lorentz angles are discussed in the next section.

\begin{tabular}{|c|c|c|c|c|c|c|c|c|}
\hline \multicolumn{4}{|c|}{ non-irradiated } & \multicolumn{5}{c|}{ irradiated with $10^{13} 21 \mathrm{MeV}^{2} \mathrm{~cm}^{2}$} \\
\hline $\begin{array}{c}U_{\text {bias }} \\
\text { in V }\end{array}$ & $\begin{array}{c}\Delta x \\
\text { in } \mu \mathrm{m} \\
(280 \mathrm{~K})\end{array}$ & $\Theta_{\text {meas }}$ & $\Theta_{\text {sim }}$ & $\begin{array}{c}U_{\text {bias }} \\
\text { in V }\end{array}$ & $\begin{array}{c}\Delta x \\
\text { in } \mu \mathrm{m}\end{array}$ & $\Theta_{\text {meas }}$ & $\begin{array}{c}\Theta_{\text {sim }} \\
\mu_{\text {low }}=1417 \\
\mathrm{~cm}^{2} / V s\end{array}$ & $\begin{array}{c}\Theta_{\text {sim }} \\
\mu_{\text {low }}=1100 \\
\mathrm{~cm}^{2} / V s\end{array}$ \\
\hline \hline 40 & $192 \pm 11$ & $33 \pm 2$ & 32 & 50 & $117 \pm 7$ & $21 \pm 1$ & $24^{\circ}$ & $20^{\circ}$ \\
100 & $170 \pm 10$ & $30 \pm 2$ & 27 & 100 & $127 \pm 7$ & $23 \pm 1$ & $29^{\circ}$ & $25^{\circ}$ \\
& & & & 150 & $126 \pm 7$ & $23 \pm 1$ & $25^{\circ}$ & $22^{\circ}$ \\
\hline
\end{tabular}

Table 1: The Lorentz shift $\Delta x$ for electrons generated at the surface for a $300 \mu \mathrm{m}$ thick sensor in a $4 \mathrm{~T}$ magnetic field as function of bias voltage for a non-irradiated sensor and an irradiated sensor. The sensor was irradiated with $21 \mathrm{MeV}$ protons up to a fluence of $10^{13} \mathrm{p} / \mathrm{cm}^{2}$. The full depletion voltage is $40 \mathrm{~V}$ for the non-irradiated sensor and $100 \mathrm{~V}$ for the irradiated sensor. $\Theta_{\text {sim }}$ was calculated from Eqs. $11.1,3.1,13$ and 3.2 


\section{Algorithm for the calculation of the Lorentz angle}

The mobility $\mu(E)$ can be parametrized by the mobility at low electric fields $\mu_{\text {low }}$ and the

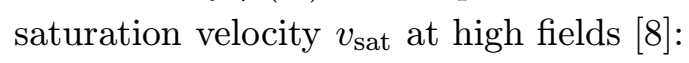

$$
\mu(E)=\frac{\mu_{\text {low }}}{\left(1+\left(\frac{\mu_{\text {low }} E}{v_{\text {sat }}}\right)^{\beta}\right)^{\frac{1}{\beta}}}
$$

Here $\beta$ is an additional constant. For holes the values are: $\beta=1.213 \cdot\left(\frac{T}{300}\right)^{0.17} ; \mu_{\text {low }}=$ $470.5 \frac{\mathrm{cm}^{2}}{V s} \cdot\left(\frac{T}{300}\right)^{-2.5} ; v_{\text {sat }}=8.37 \cdot 10^{6} \mathrm{~cm} / \mathrm{s} \cdot\left(\frac{T}{300}\right)^{0.52}$. For electrons the values are: $\beta=$ $1.109 \cdot\left(\frac{T}{300}\right)^{0.66} ; \mu_{\text {low }}=1417 \frac{\mathrm{cm} 2}{V s} \cdot\left(\frac{T}{300}\right)^{-2.2} ; v_{\text {sat }}=1.07 \cdot 10^{7} \mathrm{~cm} / \mathrm{s} \cdot\left(\frac{T}{300}\right)^{0.87}$.

The electric field is generally depending on the z-position, the bias voltage, the full depletion voltage $U_{\mathrm{depl}}$ and the thickness of the sensor:

$$
E(z)=\frac{U_{\mathrm{bias}}-U_{\mathrm{depl}}}{d}+\frac{2 \cdot U_{\mathrm{depl}}}{d} \cdot\left(1-\frac{z}{d}\right)
$$

If the sensor is fully depleted or overdepleted the average electric field (at $\mathrm{z}=\mathrm{d} / 2$ ) becomes $U_{\text {bias }} / d$. If the detector cannot be fully depleted, the field drops to zero in the undepleted region and $d$ in Eq. 3.24 corresponds to the depleted thickness with $U_{\text {bias }}=U_{\text {depl }}$. At typical bias voltages the electric field is sufficiently high to use an average mobility to calculate the Lorentz shift from Eq. 1'. Else it is easy to slice the sensor a few times and sum the Lorentz shift of each slice. Fig. measurements quite well in case of a single slice. Here we used $r_{H}=0.7(1.15)$ for holes (electrons) [2]-1].

The dependence of the mobility on the irradiation dose is still controversial. In [i $[\bar{i}]$ no significant changes were observed in the transport properties of both electrons and holes up to $0.5 \cdot 10^{14} 1 \mathrm{MeV} \mathrm{n} / \mathrm{cm}^{2}$ and a prediction is made that a fluence of at least about $10^{15} 1$ $\mathrm{MeV} \mathrm{n} / \mathrm{cm}^{2}$ is necessary to affect carrier drift mobilities significantly. In [ī $\left.]_{-1}^{1}\right]$ the mobility for both carrier types in irradiated sensors agree with those for the non-irradiated sensor within the errors for fluences up to $2 \cdot 10^{14} 1 \mathrm{MeV} \mathrm{n} / \mathrm{cm}^{2}$. In contrast a change of mobility after irradiation for holes and electrons was observed in [1] 1 in. The latter agrees with the measurements of the Lorentz shift for electrons done in Karlsruhe and measurements on the pixel detectors of ATLAS [1] irradiated ATLAS sensor could not be fully depleted, so the drift distance is not the full detector thickness, but the depleted thickness. The data was taken at $1.4 \mathrm{~T}$.

\section{Summary}

The Lorentz angle is determined by electric and magnetic fields and can be modelled by Eqs. holes and electrons, the Lorentz shift for electrons is about four times larger than for holes. The data from HERA-B test structures and the ATLAS pixel sensor could be well described 


\begin{tabular}{|c|c|c|c|c|c|}
\hline $\begin{array}{c}\text { Fluence } \\
\mathrm{n} / \mathrm{cm}^{2}\end{array}$ & $\begin{array}{c}U_{\text {bias }} \\
\text { in V }\end{array}$ & $\begin{array}{c}\text { Depl. depth } \\
\text { in } \mu \mathrm{m}\end{array}$ & $\Theta_{\text {meas }}$ & $\begin{array}{c}\Theta_{\text {sim }} \\
\mu_{\text {low }}=1417 \mathrm{~cm}^{2} / \mathrm{Vs}\end{array}$ & $\begin{array}{c}\Theta_{\text {sim }} \\
\mu_{\text {low }}=1100 \mathrm{~cm}^{2} / \mathrm{Vs}\end{array}$ \\
\hline \hline 0 & 150 & $283 \pm 6$ & $9.0 \pm 0.9$ & 8.4 & 5.7 \\
\hline $5 \cdot 10^{14}$ & 150 & $123 \pm 19$ & $5.9 \pm 1.3$ & 6.7 & 3.9 \\
$5 \cdot 10^{14}$ & 600 & $261 \pm 8$ & $2.6 \pm 0.5$ & 4.4 & 3.5 \\
\hline $10^{15}$ & 600 & $189 \pm 12$ & $3.1 \pm 1.0$ & 3.8 & 3.0 \\
$10^{15}$ & 600 & $217 \pm 13$ & $2.7 \pm 0.8$ & 3.2 & \\
\hline
\end{tabular}

Table 2: The Lorentz angle for electrons in a $280 \mu \mathrm{m}$ thick pixeldetector in a $1.4 \mathrm{~T}$ magnetic field. The measured data are taken from [i $\left.{ }_{1}^{1} 2_{1}\right]$. The full depletion voltage before irradiation is $150 \mathrm{~V}$. The algorithm used for the simulation divides the sensor into five parts, in which the mean electric field and the corresponding Lorentz shift are calculated. The averaged Lorentz angle is defined to be the arc tangent of the total Lorentz shift divided by the depletion depth. $\Theta_{\text {sim }}$ with the reduced mobility $\mu_{\text {low }}$ fits the data $\Theta_{\text {meas }}$ slightly better.

by a simple algorithm, before and after irradiation, if the reduced mobility for electrons after irradiation is taken into account.

This work was done within the framework of the RD39 Collaboration [i] $\left.{ }_{1}^{1}\right]$. We thank Iris Abt for supplying us with double sided mini-strip detectors.

\section{References}

[1] R.A. Smith, Semiconductors, Cambridge Univ. Press, 1968.

[2] Landolt-Börnstein, Numerical Data and Functional Relationships in Science and Technology, Group III, Vol. 17a, Springer Verlag, Berlin, 1982.

[3] M. Moll et al., NIM A 439 (2000) 282; RD48 Collaboration, http://rd48.web.cern.ch, ROSE/TN/2000-06, NIM A 465/1 (2001) 60.

[4] W. de Boer et al., NIM A 461 (2001), 2001; F. Röderer, Diplomarbeit, Univ. of Karlsruhe, IEKP-KA/98-24; S. Heising, Ph. D. thesis. Univ. of Karlsruhe, IEKP-KA/99-26; F. Hauler, Diplomarbeit, Univ. of Karlsruhe, IEKP-KA/2000-12;

[5] F. Hornung, A. Rimikes, Th. Schneider, High Magnetic Field facilities and Projects at the Forschungszentrum Karlsruhe, Internal Note, 1999.

[6] RD48 collaboration, http://rd48.web.cern.ch, ROSE/TN/2000-03, NIM A 466 (2001) 308.

[7] M. Huhtinen, Simulation of non-ionising energy loss and defect formation in silicon, RD48 Technical Note 2001-02, Cern(2001), submitted to NIM B.

[8] C.Canali et al., IEEE Trans. on Electron Devices, Vol.ED-22, 1045-47, 1975.

[9] V. Eremin et al., NIM A 362(1995), 338-343.

[10] T.J. Brodbeck et al., ROSE/TN/2000-09.

[11] C. Leroy et al., NIM A(1999), 90-102.

[12] M. Aleppo, NIM A 465(2001), 108-111.

[13] RD39 Collaboration, NIM A 440 (2000) 5-16 and http://rd39.web.cern.ch, RD39 Status Report CERN/LHCC 2000-010, 06-Jan-2000. 